\title{
Cornea-Based Techniques and Technology for Surgical Correction of Presbyopia
}

\author{
George O. Waring IV • Fernando Faria-Correia
}

Published online: 14 January 2014

(C) Springer Science + Business Media New York 2014

\begin{abstract}
To review current options for cornea based techniques and technologies for the surgical correction of presbyopia. Corneal inlays are an emerging and promising technology and multiple inlays are in development or in the regulatory process. Inalys may be best characterized by their mechanism of action. Thermal keratoplasty, presbyLASIK and intrastromal femtosecond ring incisions are other surgical options. Multiple options exist for the cornea based surgical correction of presbyopia.
\end{abstract}

Keywords Ophthalmology · PresbyLASIK · Corneal · Corneal-based presbyopia surgery $\cdot$ Inlays

\section{Introduction}

Presbyopia is a progressive age-related inability to accommodate, resulting in a diminished ability to focus on near objects [1•]. This ubiquitous disorder affects an estimated 1.04 billion people worldwide and results from agerelated loss of elasticity of the crystalline lens and loss of contractility of the ciliary muscle $[2 \bullet, 3 \bullet \bullet, 4,5]$.

Given this prevalence and increased interest in reduced dependence on spectacles for reading, emphasis has been placed on the development of novel treatments for the surgical correction of presbyopia. Several therapeutic modalities are now available for the refractive surgeon, depending on the patient's needs. Although traditional blended vision is still very useful, other technology has

G. O. Waring IV $(\bowtie) \cdot$ F. Faria-Correia

Department of Ophthalmology, Storm Eye Institute, Medical

University of South Carolina, Charleston, SC, USA

e-mail: georgewaringiv@gmail.com evolved which will be described in this manuscript. In addition to presbyopia-correcting intraocular lenses, several options exist for correction by refractive surgery at the corneal and scleral plane. These include: corneal inlays, conductive keratoplasty, presbyLASIK, intrastromal correction by use of femtosecond technology (INTRACOR), and scleral lasers and scleral implants. The purpose of this review is to provide an overview of cornea-based surgical procedures and devices currently in use or in development for surgical treatment of presbyopia.

\section{Corneal Inlays}

Since the original descriptions of keratophakia over half a century ago, corneal inlays have become promising treatment for surgical treatment of presbyopia. Corneal inlays have several benefits. They are removable and repositionable, because this is "addition surgery", different from "subtraction surgery" used with standard ablative techniques. Implants can also be combined with other refractive procedures and do not suffer from the risks of intraocular surgery.

Several types of implantation technique and technology are available, including both mechanical and femtosecond laser-enabled techniques for creation of pockets and flaps by use of dual interface procedures [6]. The femtosecond laser-assisted pocket technique has several advantages [6, 70.0. With this technique, most of the peripheral corneal nerves are preserved, which enables corneal sensitivity to be maintained and potentially quicker visual recovery. Theoretically, pocket procedures may be more biomechanically stable than lamellar procedures. The lamellar flap option is attractive because it enables access to a stromal bed for excimer ablation, enabling control of the refractive target and the ability to treat ametropia. The dual 
interface is another surgical technique in which the pocket is created a minimum of $120 \mu \mathrm{m}$ deeper to a previous flap, either sequentially after LASIK or from pre-existing LASIK surgery. This procedure combines the benefits of the pocket and flap techniques. All inlays are typically implanted in the non-dominant eye. The current generation of inlays available for the treatment of presbyopia may be best organized and described by the mechanism of action: changing the index of refraction, changing the corneal curvature, and small-aperture optics.

\section{Refractive Optic Inlays}

Refractive optic corneal inlays are designed to change the refractive index of the cornea, providing distance vision through a planar central zone that is surrounded by one or more rings of varying additional power for near vision.

Based on a precursor known as the InVue lens, the Flexivue Microlens (Presbia, Los Angeles, CA, USA) is a transparent, 3.0-mm-diameter hydrogel implant containing an ultraviolet blocker. It has a central $0.15 \mathrm{~mm}$ opening to facilitate fluid and nutrient flow, surrounded by a planar central zone and a refractive peripheral zone with additional power from +1.25 to $+3.5 \mathrm{D}$, in 0.25 -D steps. Depending on the additional power, the lens thickness varies between 15 and $20 \mu \mathrm{m}$. This inlay is designed to be implanted in a corneal pocket at a depth of 280-300 $\mu \mathrm{m}$.

The Presbia Flexivue inlay is currently in FDA clinical trials but is commercially available in some countries outside the US. In a prospective study of 47 presbyopic emmetropes implanted in the non-dominant eye with the Flexivue Microlens in a femtosecond laser-created corneal pocket, uncorrected near visual acuity (UCNVA) was $20 / 32$ or better for $75 \%$ of implanted eyes after 12 months (mean 20/25). Mean uncorrected distance visual acuity (UCDVA) decreased by three lines, from 20/20 to 20/50, although binocular UCDVA was not statistically significantly affected. Thirty-seven percent of patients lost one line of best-corrected distance visual acuity (BCDVA) in the operated eye; no patient lost two lines, however. There was a statistically significant decrease in mesopic and photopic contrast sensitivity at a number of spatial frequencies and an increase in higher-order aberrations in the implanted eyes. Overall patient satisfaction and spectacle independence was high, but $12.5 \%$ of patients experienced halos and glare 1 year postoperatively [7••]. Promising results were reported in a previous prospective study in which the earlier version of the inlay (InVue lens) was inserted in a corneal pocket created with a microkeratome [8•]. One year after surgery, $98 \%$ of those patients had UCNVA of 20/32 or better in the operated eye. UCDVA was $20 / 40$ or better in $93 \%$ of implanted eyes.

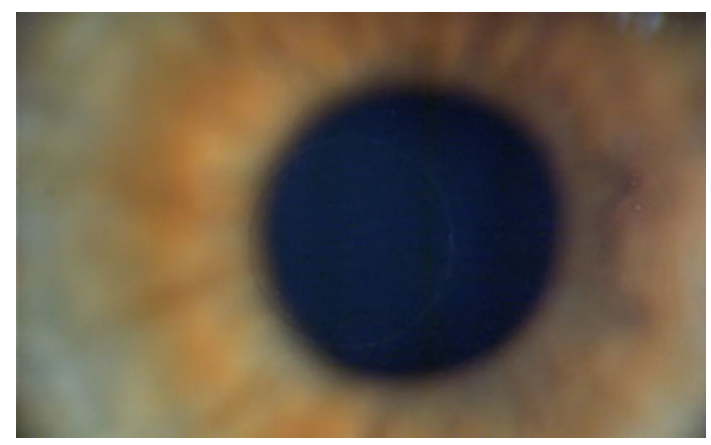

Fig. 1 Slit lamp photo of the Raindrop corneal inlay

The Icolens (Neoptics, Hunenberg, Switzerland) is a 3-mm-diameter multifocal corneal inlay which uses the peripheral zone for near vision correction and a central zone for distance vision. This lens is made of a hydrophilic acrylic hydrogel, and is the most recent inlay to be introduced into this space. Early reports from European clinical trials are promising, but no details have yet been published in the peer-reviewed literature.

\section{Cornea-Reshaping Inlays}

Here, the central anterior curvature of the cornea is increased and results in enhanced near and intermediate vision because of increased refractive power and induced spherical aberration. The Raindrop near vision inlay, formerly known as the PresbyLens or Vue+lens (ReVision Optics, Lake Forest, CA, USA) has a diameter of 1.5-2.0 $\mathrm{mm}$ and is made of hydrogel (Fig. 1). This inlay has a hyperprolate shape, $10-\mu \mathrm{m}$ thick at the edge and $30-\mu \mathrm{m}$ thick at the center. It also has no refractive power, with water content and refractive index similar to those of the native cornea. It is intended for implantation under a 130-150- $\mu \mathrm{m}$ LASIK flap or in a corneal pocket in the nondominant eye.

The Raindrop near vision inlay has a CE mark in Europe and is currently in a Phase III clinical trial in the US. Porter and colleagues described vision results from a series of hyperopic eyes with the $2.0 \mathrm{~mm}$ design implanted. There was an average UCNVA improvement of more than five lines by one month post surgery, with $78 \%$ of the implanted eyes achieving 20/25 or better. Uncorrected intermediate visual acuity (UCIVA) improved by an average of four lines, and mean distance UCDVA was 20/25 [9]. In another study, 25 hyperopic presbyopes underwent implantation of the $2.0-\mathrm{mm}$ corneal inlay combined with LASIK. After 1 month, more than $80 \%$ of these eyes achieved J1 or better UCNVA. There was also UCIVA improvement, by an average of five lines, to 20/25. 


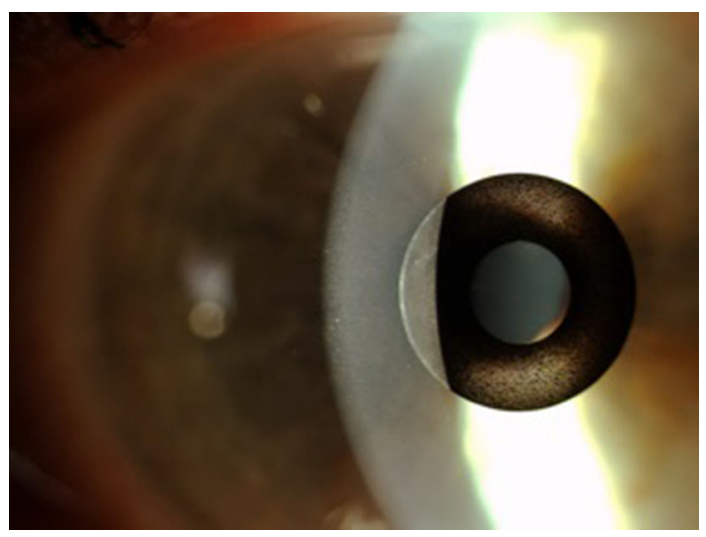

Fig. 2 Slit lamp photo of the KAMRA corneal inlay

Improvement in UCDVA averaged two lines, with one subject losing one line [10].

\section{Small-Aperture Inlays}

The Kamra Inlay (AcuFocus, Irvine, CA, USA) is an opaque inlay made of poly(vinylidene fluoride). It uses the small aperture to increase depth of field by blocking bent light rays thereby minimizing refraction (Fig. 2). The KAMRA inlay is 5- $\mu \mathrm{m}$ thick and has 8,400 laser-etched porosity holes to maintain the metabolic flow, and catabolic and hydrational homeostasis. These holes are distributed in a pseudorandom pattern to prevent diffraction issues at night. The $1.6 \mathrm{~mm}$ central annulus acts as a pinhole, and the outer diameter is $3.8 \mathrm{~mm}$. The inlay is either implanted in a lamellar pocket or under a $200-\mu \mathrm{m}$ femtosecond laser flap. Recently, a dual interface technique has been developed whereby thin-flap LASIK is performed and the inlay is then placed into a femtosecond-enabled pocket 100 microns below the LASIK flap.

Uniquely, the KAMRA inlay continuously compensates for the progressive loss of accommodative amplitude by means of improvement in depth of focus with a small aperture. In addition, in contradistinction to blended vision techniques, stereoacuity is preserved with a small-aperture inlay [16••]. A contralateral comparison of the Optical Quality Analysis System (Visiometrics, Spain) revealed a broadened defocus curve and reduced simulated retinal blur in the implanted eye (Fig. 3). The Kamra inlay is also an attractive option in several other circumstances: natural emmetropes, post-LASIK emmetropes, combined with a LASIK correction as a simultaneous or two-step procedure, and pseudophakic patients after implantation of a monofocal intraocular lens (IOL) or a phakic IOL [11••].

FDA Phase III clinical trial enrollment has been completed and this technology is commercially available in many countries outside the US, with over 20,000 inlays implanted worldwide.
Published clinical data reveal that monocular implantation of the Kamra inlay results in sustained improvement of near and intermediate vision, while maintaining good distance vision [1•]. Tomita and colleagues published results from a series of 180 eyes implanted with the current version of the Kamra inlay that also underwent simultaneous LASIK [12]. After six months, mean UCNVA for the treated eyes improved by seven lines for hyperopic eyes, six lines for emmetropic eyes, and two lines for myopic eyes; mean UCDVA improved by three lines, one line, and ten lines, respectively. All patients had binocular UCDVA of 0.00 and Log MAR 20/20 or better. Ninety-one percent of implanted eyes had uncorrected visual acuity of at least 20/32 and significant improvements in the ability to perform intermediate tasks without correction of visual acuity $[12-15 \bullet \bullet$.

Results from long-term studies with an earlier version of the Kamra inlay have also been published. Despite more recent improvements of the inlay and surgical technique, these studies showed that the mean UCNVA improved and there was no significant loss of binocular UCDVA [13••, 14•]. Despite a small loss of monocular contrast sensitivity, it remained within normal limits and binocular contrast was preserved [14•, 16॰•]. A prospective study described no significant change in stereopsis six months after implantation [16••]. After a 1-2-year follow-up prospective and interventional case series, Dexl et al. [17, 18••] reported that emmetropic presbyopes implanted with the Kamra inlay had improvement on all measures of reading performance. Other studies reveal high patient satisfaction with the Kamra inlay particularly among emmetropes and

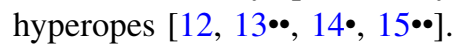

After implantation of the small-aperture inlay, patients reported a statistically significant reduction in spectacle dependence for near and intermediate tasks. The ability to perform these tasks was better under bright light conditions than in dim light $[15 \bullet \bullet, 18 \bullet \cdot]$. Recently, case reports have been published describing successful phacoemulsification with monofocal IOL implantation after KAMRA inlay implantation $[13 \bullet \bullet]$.

\section{Conductive Keratoplasty}

Conductive keratoplasty is used to correct low to moderate levels of hyperopia and astigmatism. More recently, it has also been used to induce monovision in presbyopes [19, 20]. This technique entails application of low-frequency radio waves to "shrink" collagen fibrils within the cornea; the fibrils are delivered through a fine tipped probe applied to the corneal stroma (Fig. 4). The keratoplasty tip has a $45^{\circ}$ bend proximally, to enable access to the eye over the brow or nasal region, and a $90^{\circ}$ bend distally to position the 


\section{Optical Quality Analysis System}

\section{Right Eye with KAMRA Inlay Left Eye without KAMRA Inlay}
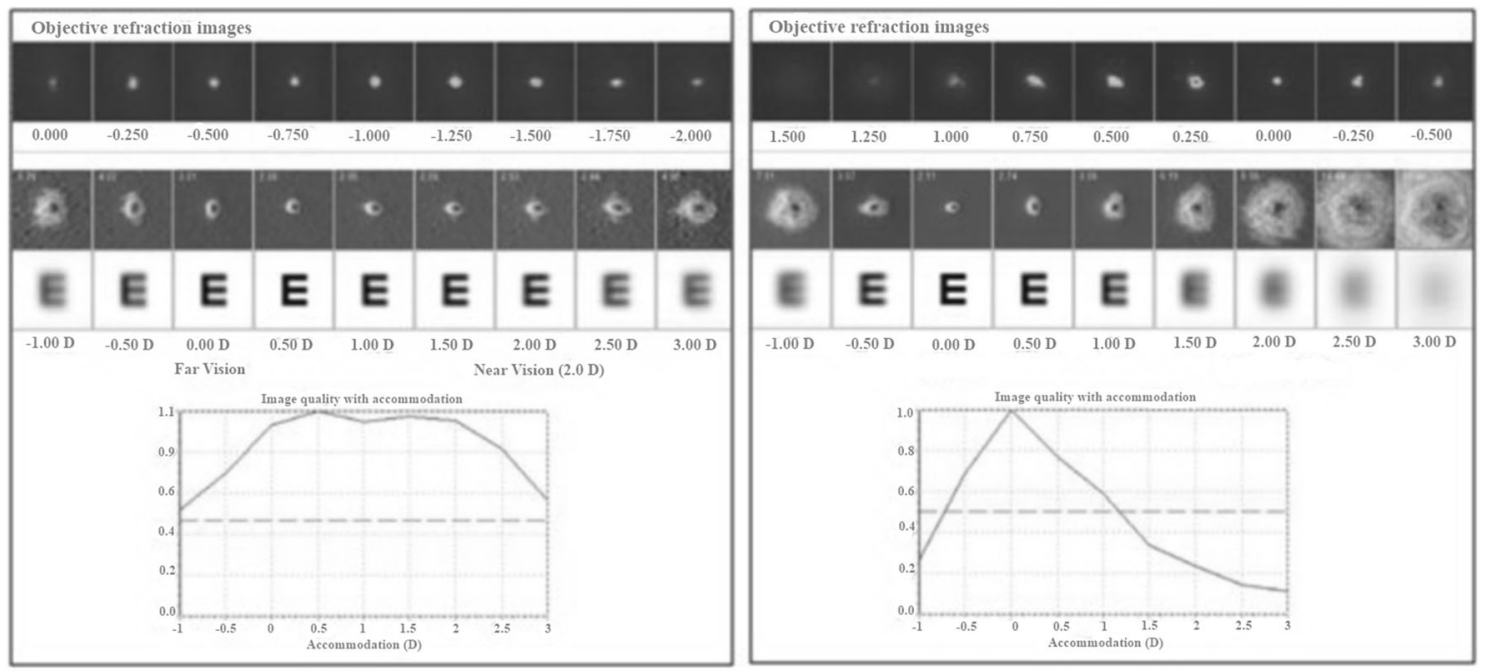

Fig. 3 Double pass wavefront demonstrating increased depth of focus with small aperture inlay compared to the fellow presbyopic eye

tip perpendicular to the corneal surface. The peripheral treatment band creates a prolate and uniform steepening of the cornea, with, therefore, increased refractive power. The number of treatment spots, which are evenly spaced in a ring pattern of 6,7 , and/or $8 \mathrm{~mm}$ in diameter, is determined by the desired correction [21]. Centering of the marker on the estimated line of sight is crucial for effective treatment. This procedure can be performed under topical anesthesia and should be efficient, aiming for $1 \mathrm{~min}$ or less per series eight spots, to avoid excessive drying of the cornea. As the collagen shrinks, the band constricts and there is a steepening of the corneal curvature central to the band. The amount of corneal steepening is controlled by treatment placement, intensity, and duration. In theory, the energy delivery is self-limiting because denaturation of collagen increases resistance to current flow [22].

One-year results from an FDA clinical trial of the use of CK to induce monovision as a treatment for presbyopia revealed $\mathrm{J} 1$ or better UCNVA for $38 \%$ (20/53) of eyes and $\mathrm{J} 3$ or better for $81 \%(43 / 53)$ of eyes treated for near vision. Binocular UCNVA of J1 or better was achieved for $47 \%(25 / 53)$ of patients and $\mathrm{J} 3$ or better for $89 \%$ (47/53) of patients. Binocular UCDVA was $20 / 20$ or better for $97 \%$ (60/62) of patients and 20/40 or better for all patients (62/62) [20].

The advantages of conductive keratoplasty over laser techniques are that it preserves the optical clarity of the visual axis and does not involve any tissue removal. Another advantage of CK is the safety of the procedure. Of 11 studies that reported safety as lines of best corrected visual acuity lost, seven of a total of 770 eyes $(1 \%)$ lost two lines of BCDVA [21-23]. All seven patients with a two-line loss of BCDVA were part of the initial FDA clinical trial treating hyperopia. The trial exceeded FDA safety standards. The low-frequency radio waves do not damage the corneal endothelium. Histologic changes are minimal in the endothelial layer, and endothelial cell counts at the corneal periphery, mid-periphery, and centrally revealed no significant change from preoperative values after 1 year of follow-up [24]. Contrast sensitivity is also spared with CK. Because the treatment zones are at the periphery of the cornea, postoperative contrast sensitivity measurements remain within normal limits [24-26].

\section{PresbyLASIK}

The idea that corneal multifocality could be achieved by excimer laser ablation resulted from the positive corneal aberrations observed for early photorefractive kerectomy patients [27]. The different approaches to creating corneal multifocality by use of LASIK are: transitional multifocality, central presbyLASIK, and peripheral presbyLASIK [28, 29]. The transitional multifocality approach has been largely abandoned. The central and peripheral techniques differ in the principle upon which they are based. Peripheral presbyLASIK depends on increasing the range of pseudoaccommodation whereas central presbyLASIK essentially creates a bifocal effect [28].

In peripheral presbyLASIK, the peripheral cornea is ablated to create a negative peripheral asphericity, which in 

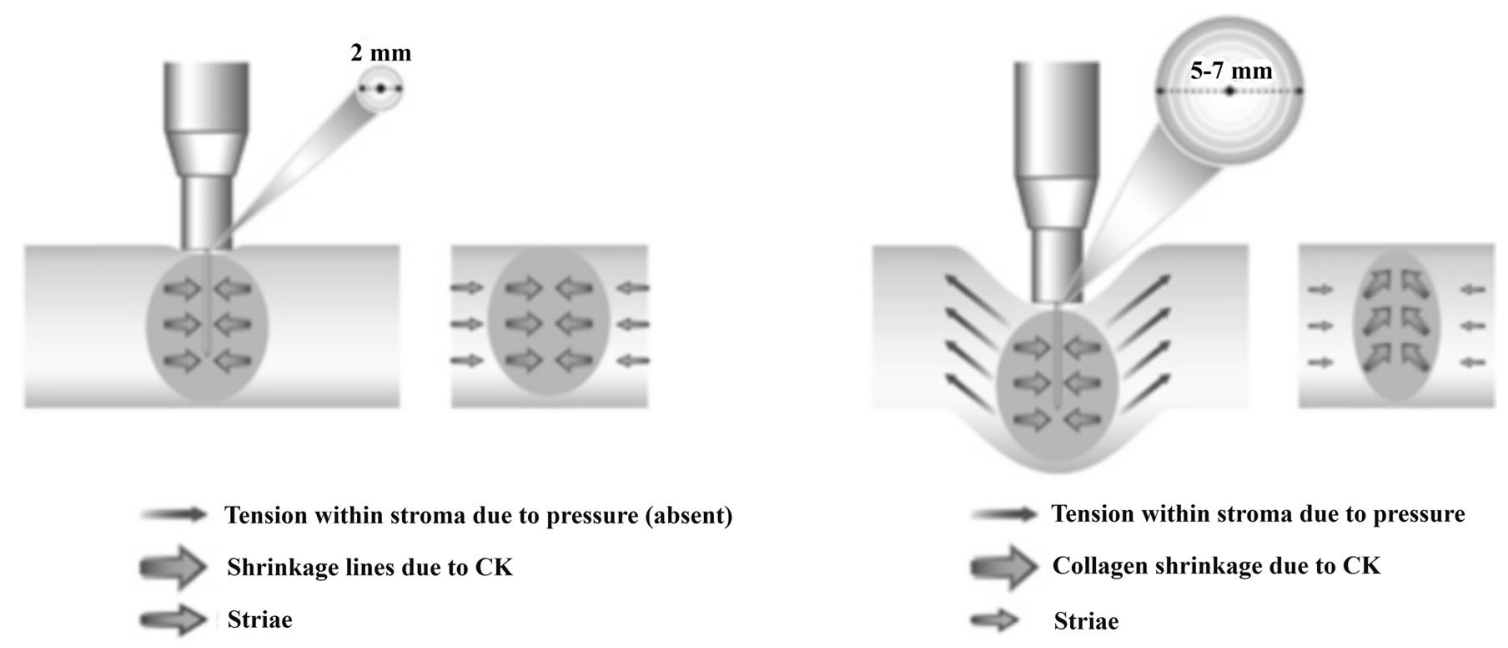

Fig. 4 Technique and mechanism of action of pressure required with CK spot placement

turn increases the depth of focus. The center of the cornea is left for distance vision whereas the peripheral cornea is used for near vision. With central presbyLASIK, a hyperpositive area for near vision in the central cornea is created, leading to a surface which functions in a similar manner to the diffractive multifocal IOLs. The largest published study of peripheral presbyLASIK included 296 eyes. When a binocularity test was performed, $98 \%$ of patients achieved UDVA of $20 / 20$ or better and $100 \%$ achieved $20 / 32$ or better; $96 \%$ achieved UCNVA of J2 and $99 \%$ could read J3 or better [29]. Similar results were obtained in other studies [30-32]. Concerning the central presbyLASIK technique, the results of UCVA were between 20/25 and $20 / 20$ for distance vision and J2 for near vision for most patients [29].

\section{Intrastromal Femtosecond Ring Incisions}

Because of the precision and the safety of the femtosecond laser, new corneal refractive surgery modalities have been developed, for example intrastromal correction of presbyopia (INTRACOR; Technolas Perfect Vision, Munich, Germany). This procedure, first described in 2009 by Ruiz et al. [33], creates a hyperprolate cornea by making a series of intrastromal corneal ring incisions [34]. Five concentric rings are cut within the area 2.0 and $4.0 \mathrm{~mm}$ from the line of sight. This should be marked before surgery by using the first Purkinje image [35].

Holzer et al. recently reported the longest follow-up results from use of this technique [35, 36]. After 18 months, median UCNVA had improved significantly from $0.7 \log$ MAR preoperatively to $0.2 \log$ MAR $(P<0.001)$ and the median corneal true net power increased significantly by $1.1-0.9$ D $(P<0.001)$. After another study, the authors reported 6 and 12-month postop outcomes [34]. After 6 months, all 83 (100\%) eyes had improved UCNVA, with minimal or no change in UCDVA. At 1 year postop, 22 eyes had UCNVA improved to J1, with improvement in mean UCDVA also. Only two (2.4\%) eyes lost two lines of CDVA after 6 months, and this was not observed for the 22 eyes seen after 1 year.

Regarding the safety of this procedure, we must remember that corneal manipulation can be associated with a risk of ectasia [37, 38]. However, Holzer et al. [36] reported stable corneal steepening and found no significant changes related with post-refractive ectasia.

\section{Conclusions}

Advances in the surgical treatment of presbyopia are occurring rapidly, and the refractive surgeon now has many options. In fact, a new subspecialty of presbyopia-correcting surgeons is emerging, and rapid innovations in technology will certainly continue. Understanding the design and mechanism of each modality will enable clinicians to match the most appropriate option to the patients' needs, anatomy, and optics, to optimize safety, efficacy, and patient satisfaction.

Acknowledgments This paper was supported in part by grants from the Research to Prevent Blindness (RPB) organization to The Department of Ophthalmology, Medical University of South Carolina.

Disclosures George Waring IV has received stock options and reimbursement for travel from Acufocus. Fernando Faria-Correia has no conflicts of interest to declare.

Human and Animal Rights and Informed Consent This article does not contain any studies with human or animal subjects performed by any of the authors. 


\section{References}

Papers of particular interest, published recently, have been highlighted as:

- Of importance

-. Of major importance

1. - Waring GO IV. Correction of presbyopia with a small aperture corneal inlay. J Refract Surg. 2011;27(11):842-5. Interim results of US IDE trial for small aperture inlay.

2. - Yilmaz OF, Bayraktar S, Agca A, et al. Intracorneal inlay for the surgical correction of presbyopia. J Cataract Refract Surg. 2008;34:1921-7. Original report for small aperture inlay.

3. • Tomita M, Tomomi K, Waring GO IV, et al. Simultaneous corneal inlay implantation and laser in situ keratomileusis for presbyopia in patients with hyperopia, myopia, or emmetropia: six-month results. J Cataract Refract Surg. 2012;38:495-506. Original and large volume report of simultaneous LASIK and inlay.

4. Pallikaris IG, Bouzoukis DI, Kymionis GD, et al. Visual outcomes and safety of a small diameter intrastromal refractive inlay for the corneal compensation of presbyopia. J Refract Surg. 2012;28:168-73.

5. Schneider J, Henderson BA. Categories of advanced technology IOLs: overview. Int Ophthalmol Clin. 2012;52:1-10.

6. Binder PS. New femtosecond laser software technology to create intrastromal pockets for corneal inlays. ARVO. 2010;51:2868.

7. • Limnopoulou AN, Bouzoukis DI, Kymionis GD, et al. Visual outcomes and safety of a refractive corneal inlay for presbyopia using femtosecond laser. J Refract Surg. 2013;29(1):12-8. Refractive corneal inlay report.

8. - Bouzoukis DI, Kymionis GD, Panagopoulou SI, et al. Visual outcomes and safety of a small diameter intrastromal refractive inlay for the corneal compensation of presbyopia. J Refract Surg. 2012;28(3):168-73. Refractive inlay report.

9. Porter T, Lang A, Holliday K, et al. Clinical performance of a hydrogel corneal inlay in hyperopic presbyopes. Invest Vis Ophthalmol Sci. 2012;53:E-abstract 4056.

10. Lang AJ, Porter T, Holliday K, et al. Concurrent use of the ReVision Optics intracorneal inlay with LASIK to improve visual acuity at all distances in hyperopic presbyopes. Invest Ophthalmol Vis Sci. 2011;52:E-Abstract 5765.

11. •• Tomita M, Kanamori T, Waring GO IV, Nakamura T, Yukawa S. Small-aperture corneal inlay implantation to treat presbyopia after laser in situ keratomileusis. J Cataract Refract Surg. 2013;39(6):898-905. Original and large volume report of inlay after LASIK.

12. Tomita M, Kanamori T, Waring GO IV, et al. Simultaneous corneal inlay implantation and laser in situ keratomileusis for presbyopia in patients with hyperopia, myopia, or emmetropia: six-month results. J Cataract Refract Surg. 2012;38(3):495-506.

13. • Yilmaz OF, Alagoz N, Pekel G, et al. Intracorneal inlay to correct presbyopia: long-term results. J Cataract Refract Surg. 2011;37:1275-81. Long term report on small aperture inlay.

14. - Seyeddain O, Hohensinn M, Riha W, et al. Small-aperture corneal inlay for the correction of presbyopia: 3-year follow-up. J Cataract Refract Surg. 2011;38(1):35-45. Long term report on small aperture inlay.

15. • Dexl AK, Seyeddain O, Riha W, et al. One-year visual outcomes and patient satisfaction after surgical correction of presbyopia with an intracorneal inlay of a new design. J Cataract Refract Surg. 2012;38(2):262-9. Original report on patient satisfaction after small aperture inlay.
16. • Linn S, Hoopes PC. Stereopsis in patients implanted with a small aperture corneal inlay. Invest Ophthalmol Vis Sci. 2012;53:E-abstract 1392. Original report on stereovision with small aperture inlay.

17. Dexl AK, Seyeddain O, Riha W, et al. Reading performance and patient satisfaction after corneal inlay implantation for presbyopia correction: two-year follow-up. J Cataract Refract Surg. 2012;38(10):1808-16.

18. $\bullet$ Dexl AK, Seyeddain O, Riha W, et al. Reading performance after implantation of a modified corneal inlay design for the surgical correction of presbyopia: 1-year follow-up. Am J Ophthalmol. 2012;153(5):994-1001. Original report on reading performance with small aperture inlay.

19. Hersh PS. Optics of conductive keratoplasty: implications for presbyopia management. Trans Am Ophthalmol Soc. 2005;103: 412-56.

20. Mendez A, Mendez Noble A. Conductive keratoplasty for the correction of hyperopia. In: Sher NA, editor. Surgery for hyperopia and presbyopia. Baltimore: Lippincott Williams \& Wilkins; 1997. p. $163-71$.

21. US FDA. Refractec ViewPoint CK System P010018. http://www. accessdata.fda.gov/scripts/cdrh/cfdocs/cfPMA/pma.cfm?id=15339 (2002). Accessed 12 May 2011.

22. Choi B, Kim J, Welch AJ, et al. Dynamic impedance measurements during radiofrequency heating of cornea. IEEE Trans Biomed Eng. 2002;49:1610-6.

23. Stahl JE. Conductive keratoplasty for presbyopia: 3-year results. J Refract Surg. 2007;23:905-10.

24. McDonald MB, Hersh PS, Manche EE, et al. Conductive keratoplasty for the correction of low to moderate hyperopia: US clinical trial 1-year results on 355 eyes. Ophthalmology. 2002;109:1978-89.

25. Pallikaris IG, Naoumidi TL, Astyrakakis NI. Long-term results of conductive keratoplasty for low to moderate hyperopia. J Cataract Refract Surg. 2005;31:1520-9.

26. Lin DY, Manche EE. Two-year results of conductive keratoplasty for the correction of low to moderate hyperopia. J Cataract Refract Surg. 2003;29:2339-50.

27. Artola A, Patel S, Schimchak P, et al. Evidence for delayed presbyopia after photorefractive keratectomy for myopia. Ophthalmology. 2006;113:735-41.

28. Alio' JL, Amparo F, Ortiz D, et al. Corneal multifocality with excimer laser for presbyopia correction. Curr Opin Ophthalmol. 2009;20:264-71.

29. Reinstein DZ, Couch DG, Archer TJ. LASIK for hyperopic astigmatism and presbyopia using micro-monovision with the Carl Zeiss Meditec MEL80. J Refract Surg. 2009;25:37-58.

30. Cantu' R, Rosales MA, Tepichin E, et al. Advanced surface ablation for presbyopia using the Nidek EC-5000 laser. J Refract Surg. 2004;20:S711-3.

31. Cantu' R, Rosales MA, Tepichı'n E, et al. Objective quality of vision in presbyopic and nonpresbyopic patients after pseudo accommodative advanced surface ablation. J Refract Surg. 2005; 21:S603-5

32. Pinelli R, Ortiz D, Simonetto A, et al. Correction of presbyopia in hyperopia with a center-distance, paracentral-near technique using the Technolas 217z platform. J Refract Surg. 2008;24: 494-500.

33. Ruiz LA, Cepeda LM, Fuentes VC. Intrastromal correction of presbyopia using a femtosecond laser system. J Refract Surg. 2009;25:847-54

34. Guedj T, Danan A, Lebuisson DA. In-vivo architectural analysis of intrastromal incisions after INTRACOR surgery using Fourierdomain OCT and Scheimpflug imaging. J Emmetropia. 2011;2: $85-91$. 
35. Holzer MP, Mannsfeld A, Ehmer A, et al. Early outcomes of INTRACOR femtosecond laser treatment for presbyopia. J Refract Surg. 2009;25:855-61.

36. Holzer MP, Menassa N, Fitting A, et al. Visual outcomes and corneal changes after intrastromal femtosecond laser correction of presbyopia. J Cataract Refract Surg. 2012;38: 765-73.
37. Randleman JB, Woodward M, Lynn MJ, et al. Risk assessment for ectasia after corneal refractive surgery. Ophthalmology. 2008; 115:37-50.

38. Saad A, Grise-Dulac A, Gatinel D. Bilateral loss in the quality of vision associated with anterior corneal protrusion after hyperopic LASIK followed by intrastromal femtolaser-assisted incisions. J Cataract Refract Surg. 2010;36:1994-8. 